\title{
An electrophysiological signature for proactive interference resolution in working memory
}

\author{
Yingchun Du ${ }^{\text {a,d }}$, Zhuangwei Xiao ${ }^{a}$, Yan Song ${ }^{b}$, Silu Fan ${ }^{c}$, Renhua Wu ${ }^{\text {d,* }}$, John X. Zhang a,e,* \\ a Guangdong Province Key Laboratory of Medical Molecular Imaging, Shantou University Medical College, Shantou, China \\ b State Key Laboratory of Cognitive Neuroscience and Learning, Beijing Normal University, Beijing, China \\ c Graduate School of the Chinese Academy of Sciences, Beijing, China \\ d Department of Medical Imaging, The Second Affiliated Hospital of Shantou University Medical College, Shantou, China \\ e Department of Psychology, Chinese University of Hong Kong, Hong Kong, China
}

\section{A R T I C L E I N F O}

\section{Article history:}

Received 19 December 2007

Received in revised form 17 March 2008

Accepted 17 March 2008

Available online 26 March 2008

\section{Keywords:}

Proactive interference

ERP

N2

Familiarity

Context

\begin{abstract}
A B S T R A C T
We used event-related potentials (ERPs) to study the temporal dynamics of proactive interference in working memory. Participants performed a Sternberg item-recognition task to determine whether a probe was in a target memory set. Familiar negative probes were found to be more difficult to reject than less familiar ones. A fronto-central N2 component peaking around $300 \mathrm{~ms}$ post-probe-onset differentiated among target probes, familiar and less familiar non-target probes. The study identifies N2 as the ERP signature for proactive interference resolution. It also indicates that the resolution process occurs in the same time window as target/non-target discrimination and provides the first piece of electrophysiological evidence supporting a recent interference resolution model based on localization data [Jonides, J., Nee, D.E., 2006. Brain mechanisms of proactive interference in working memory. Neuroscience 139, 181-193].
\end{abstract}

(c) 2008 Elsevier B.V. All rights reserved.

\section{Introduction}

Proactive interference (PI) occurs when memories acquired earlier impair recall or recognition of memories acquired more recently (Keppel and Underwood, 1962). It used to be a central topic in memory research, extensively studied both in short-term and long-term memory. As a determinant of working memory capacity, it is also important to higher cognitive functions that depend on working memory (Just and Carpenter, 1999).

Monsell (1978) designed a "recent-probe" paradigm to effectively measure and study proactive interference in working memory. In this paradigm, participants perform a Sternberg memory-scanning task to determine if a probe is in a set of target items they hold in mind. Critically, the probe familiarity is manipulated to be either high or low, depending on whether or not it is drawn from recent previous trials'

\footnotetext{
is This research was supported by grants from the National Natural Science Foundation of China (\#30570606, \#30670702) and Guangdong Natural Science Foundation of China (\#2010434), and a direct grant from Chinese University of Hong Kong (\#2020911).

* Corresponding authors. Zhang is to be contacted at Guangdong Province Key Laboratory of Medical Molecular Imaging, Medical College of Shantou University, 51 \# Changping Road, Shantou, China. Wu, The Second Affiliated Hospital, Medical College of Shantou University, Shantou, China.

E-mail addresses: rhwu@stu.edu.cn (R. Wu), jxzhang@cuhk.edu.hk (J.X. Zhang).
}

memory sets. PI is observed as a delayed response in rejecting familiar non-target probes, relative to less familiar non-target probes.

With this paradigm, Jonides et al. (1998) made the first attempt to examine proactive interference with modern brain imaging techniques and identified the left inferior frontal gyrus as a neural substrate for such interference resolution. This finding has been replicated in several later studies either with the same paradigm (D'Esposito et al., 1999; Bunge et al., 2001; Thompson-Schill et al., 2002; Mecklinger et al., 2003; Postle and Brush, 2004; Badre and Wagner, 2005), or with a different one involving directed-forgetting (Zhang et al., 2003a).

Recently, Jonides and Nee (2006) reviewed the current imaging evidence and proposed a biased-competition model for proactive interference resolution. In this model, a probe is classified as a target or a non-target based on evaluation of attributes associated with its representation, including familiarity codes and context codes. High familiarity non-targets elicit interference in this evaluation process due to attribute inconsistency, as familiarity codes call for a positive response but context codes for a negative response. The interference is resolved through biasing evaluation to weight more on the appropriate context codes and less on the inappropriate familiarity codes.

The present study was intended to test this model with the high temporal resolution event-related potential (ERP) technique. Specifically, we examined a central claim of the model that proactive interference is resolved in the same evaluation process that distinguishes between positive and negative probe items. To support this claim, one would 
predict the electrophysiological correlate of proactive interference to occur in the same time window when the positive and negative probes are differentiated. It would be difficult, if not impossible, to test such a prediction with function MRI at its present temporal resolution.

Our task followed that in Zhang et al. (2003a) where participants held an initial set of four letters in mind and were then instructed to ignore two of them and remember the remaining two as the target set. Later, they judged whether or not a probe was in the target set. The probe was in the memory set for the Yes trials (requiring a positive response), but not in the set for the No or Lure trials (both requiring a negative response). The Lure condition, with probes drawn from recently ignored letters, resembles the "high-familiarity recentnegative" condition in Jonides et al. (1998). For the No condition, the probes did not appear in the initial memory set, making this condition resemble their "low-familiarity non-recent negative" condition. Proactive interference was indexed as delayed response to probes in the Lure condition than in the No condition, as shown in Zhang et al. (2003a).

Several ERP studies using variants of the Sternberg task showed that there are at least two components sensitive to the distinction between Yes and No trials. Kramer et al. (1991) reported a delayed and smaller parietal P3 for negative probes relative to positive probes, as well as a larger frontal negativity (N340). The same effect on P3 amplitude was also found in our previous study (Zhou et al., 2004) which, in addition, documented without further analysis an enhanced anterior $\mathrm{N} 2$ for the negative trials relative to positive trials. This negative deflection seems to resemble a N270 component that Wang and colleagues found to be associated with mismatch detection under a variety of task settings (Wang et al., 2004; Zhang et al., 2003b).

It is more difficult to identify a priori the ERP component that would be sensitive to proactive interference in working memory as the present study seems to be the first one of its kind. In long-term memory research, we have only found two ERP studies on proactive interference, which used the paired-association paradigm and reported a more pronounced positive deflection in the P3 latency range for the interference condition, relative to the control condition (Uhl et al., 1990; Rößner et al., 1999).

\section{Methods}

\subsection{Participants}

Eighteen college students (mean age $=23.2$ years, 8 male) from Beijing Normal University (Beijing, China) were recruited. All were right-handed with normal or corrected-to-normal vision. None of them reported any neurological or psychiatric diseases. Informed consent was obtained in accordance with guidelines from the Institute of Psychology, the Chinese Academy of Sciences, Beijing. Data from four participants were discarded because of excessive number of trials contaminated by eye blinks and other artifacts.

\subsection{Stimuli and procedure}

Participants were seated in a dimly lit and sound-attenuated room. All visual stimuli were in black with a white background, presented on a computer monitor that was about $1 \mathrm{~m}$ away, subtending a visual angle of $7.7^{\circ} \times 7.2^{\circ}$. Participants were instructed to remain relaxed and to minimize motion-related artifacts throughout the experiment.

All participants completed 7 blocks, each with 40 trials. The first block served for practice and was excluded from data analysis. The procedure mainly followed that of Zhang et al. (2003a). As in Fig. 1, each trial started with a 2000-ms preparation screen with a small central square dot, followed by a study display presented for $1000 \mathrm{~ms}$. A 500-ms fixation cross display was then shown, followed by a 500-ms cue display. There was another 1500 -ms fixation display

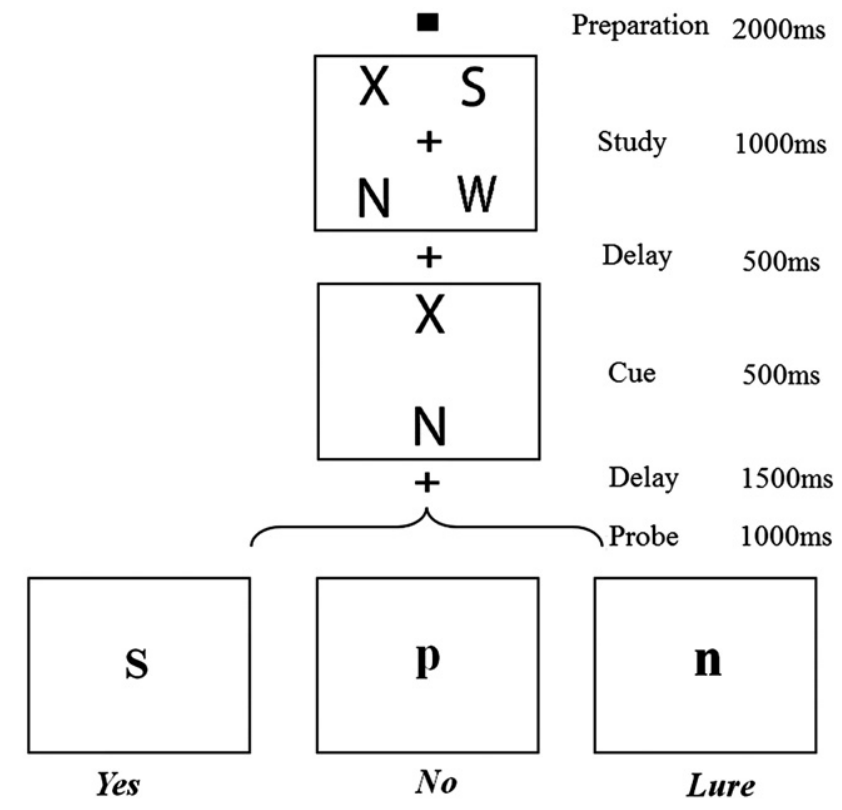

Fig. 1. Illustration of the task and conditions with a sample trial. Participants saw four consonant letters ('X N S W') in the study display and were then cued to ignore two of them (' $\mathrm{X} N$ ') and focus on remembering the rest two ('S W') as the memory set. They responded to a probe by deciding whether it was a member of the memory set. In the Yes condition, the probe ('s') was in the memory set, in the No condition, it ('p') was not. In the Lure condition, the probe ('n') was not in the memory set but drawn from the recently ignored cue letters.

after cue offset and before probe onset. The probe letter would be turned off as soon as participants responded or at the end of a 1000 -ms response window. Following probe offset, a fixation cross was displayed for $1000 \mathrm{~ms}$ before the next trial started.

Participants were told to remember the four letters in the study display (e.g., 'X N S W') before they saw the two cue letters (e.g., 'X N'), which were always the same as either the left or the right two study letters with equal probability. Upon cue onset, they were to ignore the cue letters and hold only the remaining two letters (e.g., 'S W') as the final memory set. They then judged if the probe letter (e.g., ' $n$ ') was in the final memory set or not. Both response speed and accuracy were emphasized. Each participant used one thumb to press a button indicating a Yes response and a different thumb to press a different button indicating a No response. The mapping between Yes/No responses was counterbalanced across subjects.

Drawn from a pool of 21 consonants, all letters were presented in upper-case except the probe letter which was in lower-case. For Yes trials, the probe was randomly picked from the two letters in the final memory set. For No trials, the probe was not among the four study letter. For Lure trials, the probe was randomly picked from the cue letters. All letters in any trial would not repeat in the immediately preceding or following trials. There were 20 Yes trials, 10 No trials and 10 Lure trials in each block, randomly intermixed.

\subsection{Recording system and data analysis}

EEG was recorded from the scalp through 64 non-polarizable $\mathrm{Ag} / \mathrm{AgCl}$ sintered electrodes in pre-configured caps, with a $500 \mathrm{~Hz}$ sampling rate using the Neuroscan EEG system. The sites of electrodes followed the extended 10-20 convention. Mean of both mastoids was used as reference and $\mathrm{AFz}$ as ground. Bandwidth ranged from 0.05 to $40 \mathrm{~Hz}$. The horizontal EOG was recorded at the outer canthi of both eyes and the vertical EOG recorded between supraorbit and suborbit of the left eye. Impedance in all electrodes was below $8 \mathrm{k} \Omega$. Data inspection and artifact rejection were performed offline. Eye movement artifacts were removed using regression-based weighting 
coefficients. Epochs with amplitudes exceeding $\pm 80 \mu \mathrm{v}$ in any channel were excluded with an automated procedure. Only waveforms from behaviorally correct trials were analyzed. ERPs were calculated offline and separately for each of the three types of trials with 1600 ms epochs starting 200 ms before probe onset. Mean reaction time (RT) and error rate were analyzed using a repeatedmeasure one-way ANOVA with trial type as the factor. Only correct trials were included in RT analysis.

\section{Results}

\subsection{Behavioral data}

The mean RTs averaged over all fourteen valid participants were $588 \mathrm{~ms}(\mathrm{SD}=3.8), 612 \mathrm{~ms}(\mathrm{SD}=5.3)$, and $659 \mathrm{~ms}(\mathrm{SD}=5.4)$ for the Yes, No, and Lure conditions, respectively. The corresponding error rates were $7.3 \%(\mathrm{SD}=0.5), 2.5 \%(\mathrm{SD}=0.8)$, and $5.0 \%(\mathrm{SD}=0.8)$. ANOVA on RT revealed a significant main effect of trial condition $(F(2,26)=35.72$, $p<0.0001)$. Post-hoc comparisons indicated that No trials were slower than Yes trials (612 vs. $588 \mathrm{~ms}, t(13)=3.16, p<0.005$ ), and Lure trials were slower than No trials (659 vs. $612 \mathrm{~ms}, t(13)=5.80, p<0.0001$ ). For error rates, there was a significant main effect of trial condition $(F(2,26)=12.81, p<0.01)$, with Yes trials being less accurate than No trials $(7.3 \%$ vs. $2.5 \%, t(13)=5.0, p<0.0001)$ and Lure trials being less accurate than No trials ( $5.0 \%$ vs. $2.5 \%, t(13)=2.27, p<0.05)$.

\subsection{Electrophysiological data}

Grand average potentials over fourteen participants, as depicted in Fig. 2, revealed in most electrode sites a sequence of positive and negative deflections, which we identified as the N1, P2, N2, and P3 components. As modulations of these components by trial condition, central to our interest, were mostly seen in the fronto-central regions, we conducted ANOVA on representative electrodes in these regions (except for P3, see below). Note the anterior N1 and P2 components seen here may be different from those observed in occipital regions in the literature.

Repeated-measure ANOVAs with Greenhouse-Geisser correction were performed on the amplitudes of N1, P2, and N2, with trial type
(Yes, Lure, and No), laterality (left hemisphere, midline, and right hemisphere), and gradient (frontal: F3, FZ, F4; fronto-central: FC3, FCz, FC4; and central: C3, Cz, C4) as factors. Results related to the laterality factor were omitted as it did not interact with the No vs. Lure comparison that defined proactive interference as our central interest. For P3, both the laterality and gradient factors were omitted. Examining only the trial type factor, repeated-measure one-way ANOVAs were conducted on P3 amplitude and latency using six parietal electrodes $(\mathrm{Pz}, \mathrm{P} 3 / 4, \mathrm{CPz}$, and $\mathrm{CP} 3 / 4)$ where this component is known to be most evident. All amplitudes were measured relative to the pre-stimulus baseline.

3.3. $N 1$

The mean amplitude of $\mathrm{N} 1$ was measured in the $80-120 \mathrm{~ms}$ time window. There were only two main effects on trial type and gradient $(F(2,26)=6.60, p<0.01 ; F(2,26)=12.53, p<0.001)$ but not any interaction effects $(F s<1)$. Post-hoc comparisons showed reduced N1 in Lure trials relative to No trials ( -0.51 vs. $-1.56 \mu \mathrm{v}, t(13)=4.28, p<0.001$ ) but no difference between Yes and Lure trials ( -1.01 vs. $-0.51 \mu v, t(13)=1.48$, $p>0.1$ ), between Yes and No trials (-1.01 vs. $-1.56 \mu \mathrm{v}, t(13)=2.08$, $p=0.06)$.

\section{4. $P 2$}

The positively going waveform following N1 was identified as P2 whose mean amplitude was measured from the 150-200 ms time window. There was a main effect for trial type $(F(2,26)=4.83, p<0.05)$ and for gradient $(F(2,26)=9.1, p<0.005)$ but the two did not interact $(F<1)$. Lure trials showed a larger amplitude than did Yes trials and No trials (5.61 vs. $4.89 \mu \mathrm{v}, t(13)=2.51, p<0.05 ; 5.61$ vs. $4.55 \mu \mathrm{v}, t(13)=3.16$, $p<0.01$ ), but there was no difference between Yes and No trials (4.89 vs. $4.55 \mu \mathrm{v}, t(13)=0.84, p>0.1)$.

\subsection{N2}

N2 was identified as the most negatively going peak in the latency range of 250-300 ms with mean amplitude averaged over the 200-350 ms time window. There was a main effect for trial type
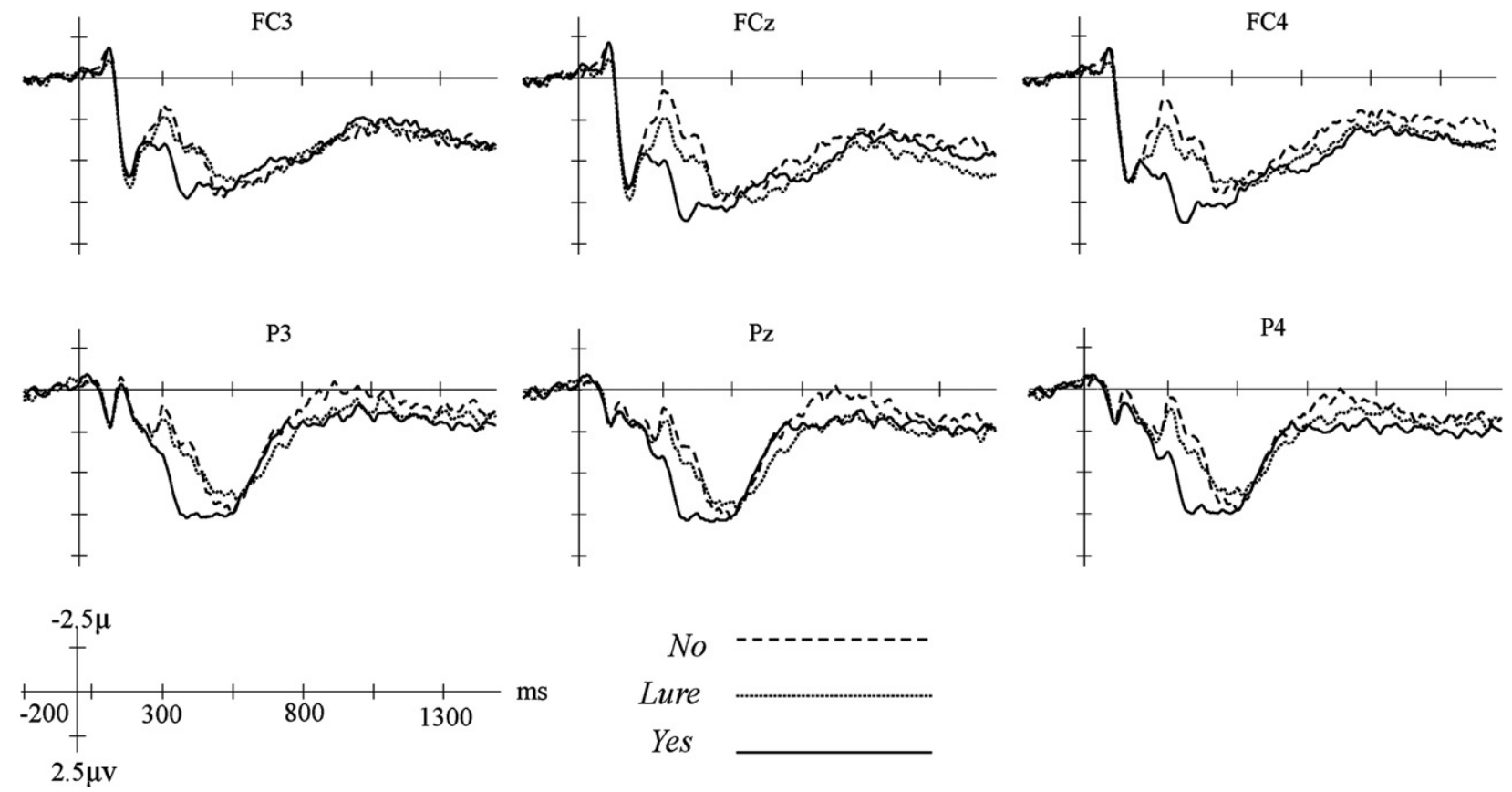

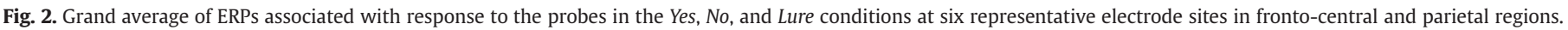


$(F(2,26)=11.03, p<0.0001)$ but not for gradient or their interaction $(F s<1)$. N2 showed a larger negative shift for No trials than for Lure and Yes trials ( 2.31 vs. $3.64 \mu \mathrm{v}, t(13)=3.40, p<0.005 ; 2.31$ vs. $4.70 \mu \mathrm{v}, t(13)=$ 3.73, $p<0.005)$. Difference between N2 amplitude for Lure and Yes trials also reached significance (3.64 vs. $4.70 \mu v, t(13)=2.28, p<0.05$ ).

\section{6. $P 3$}

P3 peak was defined as the maximum positive point in the waveform during the 300-600 ms period and its mean amplitude was averaged over a $120-\mathrm{ms}$ time window centered at the peak. The trial type effect was significant on P3 latency $(F(2,26)=10.63, p<0.001)$ but not on P3 amplitude $(F(2,26)=1.63, p>0.1)$. The latency was significantly shorter for Yes trials than for both $N o$ (407 vs. $503 \mathrm{~ms}, t(13)=$ $3.98, p<0.005$ ) and Lure trials ( 407 vs. $482 \mathrm{~ms}, t(13)=3.33, p=0.005$ ).

\section{Discussion}

The behavioral results replicated the Zhang et al. (2003a) study in finding a clear proactive interference effect, i.e., response to probes in the Lure condition was significantly slower than in the No condition ( 659 vs. $612 \mathrm{~ms}$ ). Error rate effect showed a consistent pattern (5.0\% vs. $2.5 \%)$. That the Yes responses were significantly faster than the No responses (588 vs. $612 \mathrm{~ms}$ ) is typical in the Sternberg task literature, often explained with a serial memory scanning process (Sternberg, 1966). The higher error rate for the Yes trials relative to the No trials (7.3\% vs. 2.5\%) may result from confusion between the study set and the cue set caused by retroactive interference. Such confusion, however, would have had little effect on the No trials where the probe was out of either set.

The ERP data shows that, shortly after the probe onset, the brain already differentiated between the No and the Lure trials, with an amplitude reduction in $\mathrm{N} 1$ around $100 \mathrm{~ms}$ and an amplitude increase in P2 around $175 \mathrm{~ms}$. Such a positive shift of the N1-P2 complex may be related to attentional suppression of Lure probes that were actively ignored just recently and became no-longer relevant, as found in some previous studies (Alho et al., 1987; Filipović et al., 2000). That there was no significant difference between the high-familiarity Yes and the low-familiarity No trials suggests an absence of familiarity effect at this early stage. This interpretation needs confirmation in future research as it implies that the Lure trials should differ from the Yes trials as well. However, this was statistically true only for P2 but not for N1.

As expected, both N2 and P3 showed differentiation between the Yes and the No trials, and are thus interpreted to be involved in classifying probes as target or non-target. The N2 component, prominent in fronto-central areas, showed a significantly larger amplitude for the No condition than for the Yes condition, as in Kramer et al. (1991), Wang et al. (2004), and Zhou et al. (2004) described in the introduction. Its latency was $300 \mathrm{~ms}$, in the same range of the N270, N350 reported in these studies.

The P3 component, prominent in parietal area where it is typically observed, peaked significantly later in the No condition than in the Yes condition. This P3 effect was only on latency, therefore similar but not identical to those in other studies. While Kramer et al. (1991) found P3 both smaller and delayed for negative trials, Zhou et al. (2004) only found an amplitude effect. It remains to see which factors are important for a latency effect and which for an amplitude effect. One such factor may be task complexity. The Zhou et al. (2004) study, although not focusing on Yes/No differences, adopted a Sternberg task highly comparable to the present one. Both used letter sets presented visually and simultaneously. Both used a memory set of two letters and a target-probe delay interval around $1500 \mathrm{~ms}$. Therefore, the different effects observed may be due to the introduction of Lure probes in the present experiment which could have affected processing of the No probes and produced a processing delay.
The novel and more critical result is that the N2 amplitude was significantly smaller for the Lure trials than for the No trials. This suggests that the N2 component was sensitive to the distinction between these two types of negative trials and therefore related to proactive interference. The ERP waveforms for the Lure and the No trials converged totally in P3, indicating that PI was fully resolved within the time window of N2. Examination of the stimulus-locked Lateralized Readiness Potential (LRP) results did not reveal any significant differences in onset time (an index for response competition) between these two conditions, excluding the possibility that PI arose at the level of response selection.

Briefly, N2, while sensitive to target/non-target discrimination, was also indicative of PI resolution. This pattern of results supports the biased-competition model (Jonides and Nee, 2006) described in the introduction which predicts that the PI resolution involves the same pre-response evaluation process for target/non-target discrimination.

The model also accounts for the result that the $\mathrm{N} 2$ amplitude for the Lure trials was in between that of the No and Yes trials. According to the model, the probe was evaluated based on two memory attributes, familiarity and context codes. The Yes probes, eliciting a smaller N2, were associated with high familiarity and appropriate context codes, and the No probes, eliciting a larger N2, were associated with low familiarity and inappropriate context codes. The Lure probes, being half like the Yes probes (high familiarity) and half like the No probes (appropriate context codes), was evaluated under the influence of the two attributes towards opposite directions and therefore elicited a compromised N2 with intermediate amplitude. As times went on and the evaluation process was biased to weight more on the correct context codes, the waveform of the Lure trials eventually merged with that of the No trials, indicating complete disregard of influence from familiarity and the resolution of PI.

Although we explain the results with the evaluation process to resolve code incompatibility, the N2 effect is also predicted by the degree of mismatch. That is, the N2 amplitude gets increasingly larger from the Yes probes matching on both code recency (i.e., familiarity) and context relevance, the Lure probes matching on recency but not context, to the No probes matching on neither. This suggests that our N2 may be functionally identified with the anterior N270 reported by Wang and colleagues (Wang et al., 2004; Zhang et al., 2003b), which is elicited by non-targets and considered to reflect mismatch detection. In contrast, our N2 should be distinguished from a 'N2c' component in other studies (e.g., Ritter et al., 1979; Hillyard and Münte, 1984) which has posterior scalp distribution and is primarily elicited by targets or target features (see Folstein and Van Petten, 2008 for a detailed discussion).

One issue to be addressed in future research is to examine how to relate the present interpretation of N2 to another account of N2 as index for inhibition (Heil et al., 2000; Ramautar et al., 2006) or conflict-monitoring (Nieuwenhuis et al., 2003; Yeung and Matthew, 2004). In those contexts, a larger N2 is typically observed for conditions involving inhibition or conflict. Such a condition in the present study, the Lure condition, however, elicited a smaller N2. Another issue is to examine whether our results are specific to verbal materials as proactive interference can be modality-dependent (Leung and Zhang, 2004).

\section{Conclusion}

The present ERP study examined the temporal profile of proactive interference in working memory using a Sternberg item-recognition task and identified a fronto-central N2 component as its electrophysiological correlate. With similar scalp distribution and eliciting conditions, this component resembles a previously reported N270 component considered to reflect mismatch detection. The data show that proactive interference occurs in the same time window where targets and non-targets are discriminated and its resolution seems to 
involve biased-competition between two kinds of memory attributes. Such results are consistent with and therefore provide the first piece of ERP evidence for a model of proactive interference resolution (Jonides and Nee, 2006) that has been primarily developed from brain imaging data focusing on functional localization.

\section{References}

Alho, K., Tottola, K., Reinikainen, K., Sams, M., Näätänen, R., 1987. Brain mechanisms of selective listening reflected by event-related potentials. Electroencephalogr. Clin. Neurophysiol. 68, 458-470.

Badre, D., Wagner, A.D., 2005. Frontal lobe mechanisms that resolve proactive interference. Cereb. Cortex 15, 2003-2012.

Bunge, S.A., Ochsner, K.N., Desmond, J.E., Glover, G.H., Gabrieli, J., 2001. Prefrontal regions involved in keeping information in and out of mind. Brain 124, 2074-2086.

D'Esposito, M., Postle, B., Jonides, J., Smith, E.E. 1999. The neural substrate and tempora dynamics of interference effects in working memory as revealed by event-related functional MRI. Proc. Natl. Acad. Sci. 96, 7514-7519.

Filipović, S.R., Jahanshahi, M., Rothwell, J.C., 2000. Cortical potentials related to the nogo decision. Exp. Brain Res. 132, 411-415.

Folstein, J.R., Van Petten, C., 2008. Influence of cognitive control and mismatch on the N200: a review. Psychophysiology 45, 152-170.

Heil, M., Osman, A., Wiegelmann, J., Rolke, B., Hennighausen, E., 2000. N200 in the Eriksen task: inhibitory executive processes? J. Psychophysiol. 14, 218-225.

Hillyard, S.A., Münte, T.F., 1984. Selective attention to color and location: an analysis with event-related brain potentials. Percept. Psychophys. 36, 185-198.

Jonides, J., Nee, D.E., 2006. Brain mechanisms of proactive interference in working memory. Neuroscience 139, 181-193.

Jonides, J., Smith, E.E., Marshuetz, C., Koeppe, R.A., 1998. Inhibition in verbal working memory revealed by brain activation. Proc. Natl. Acad. Sci. 95, 8410-8413.

Just, M.A., Carpenter, P.A., 1999. A capacity theory for comprehension. Psychol. Rev. 99, 122-149.

Keppel, G., Underwood, B.J., 1962. Proactive inhibition in short-term retention of single items. J. Verbal Learn. Verbal Behav. 1, 153-161.

Kramer, A.F., Strayer, D.L., Buckley, J., 1991. Task versus component consistency in the development of automatic processing: a psychophysiological assessment. Psychophysiology 28, 425-437.
Leung, H.C., Zhang, J.X., 2004. Frontal and parietal activation in discrimination of target/non-target stimuli in spatial working memory. Neurolmage 23,1013-1019.

Mecklinger, A., Weber, K., Gunter, T.C., Engle, R.W., 2003. Dissociable brain mechanisms for inhibitory control: effects of interference content and working memory capacity. Cogn. Brain Res. 18, 28-38.

Monsell, S., 1978. Recency, immediate recognition, and reaction time. Cogn. Psychol. 10, 465-501.

Nieuwenhuis, S., Yeung N., Van den Wildenberg, W., Ridderinkhof, K.R., 2003. Electrophysiological correlates of anterior cingulate function in a go/no-go task: effects of response conflict and trial type frequency. Cogn. Affect. Behav. Neurosci. 3, $17-26$.

Postle, B.R., Brush, L.N., 2004. The neural bases of the effects of item non-specific proactive interference in working memory. Cogn. Affect. Behav. Neurosci. 4, 379-392.

Ramautar, J.R., Kok, A., Ridderinkhof, K.R., 2006. Effects of stop-signal modality on the N2/P3 complex elicited in the stop-signal paradigm. Biol. Psychol. 72, 96-109.

Ritter, W., Simson, R., Vaughan, H.G., Friedman, D., 1979. A brain event related to the making of a sensory discrimination. Science 203, 1358-1361.

Rößner, P., Rockstroh, B., Cohen, R., Wagner, M., Elbert, T., 1999. Event-related potential correlates of proactive interference in schizophrenic patients and controls. Psychophysiology 36, 199-208.

Sternberg, S., 1966. High-speed scanning in human memory. Science 153, 652-654.

Thompson-Schill, S.L., Jonides, J. Marshuetz, C. Smith, E.E., D'Esposito, M., Kan, I.P. Knight, R.T., Swick, D., 2002. Effects of frontal lobe damage on interference effects in working memory. Cogn. Affect. Behav. Neurosci. 2, 109-120.

Uhl, F., Franzen, P., Serles, W., Lindinger, G., Deecke, L., 1990. Anterior frontal cortex and the effect of proactive interference in paired associate learning: a DC-potential study. J. Cogn. Neurosci. 2, 373-382.

Wang, Y., Cui, L., Wang, H., Tian, S., Zhang, X., 2004. The sequential processing of visual feature conjunction mismatches in the human brain. Psychophysiology 41, 21-29.

Yeung, N., Matthew, M.B., 2004. The neural basis of error detection: conflict monitoring and the error-related negativity. Psychol. Rev. 111, 931-959.

Zhang, J.X., Leung, H.C., Johnson, M.K., 2003a. Frontal activations associated with accessing and evaluating information in working memory: an fMRI study. Neurolmage 20, 1531-1539.

Zhang, X., Wang, Y., Li, S., Wang, L., 2003b. Event-related potential N270, a negative component to identification of conflicting information following memory retrieval. Clin. Neurophysiol. 114, 2461-2468.

Zhou, B., Zhang, J.X., Tan, L.H., Han, S.H., 2004. Spatial congruence in working memory: an ERP study. NeuroReport 15, 2795-2799. 